\title{
Anxiety responses to maximal exercise testing
}

\author{
Patrick J. O'Connor, Steven J. Petruzzello, Karla A. Kubitz and Tracey L. Robinson \\ Exercise and Sport Research Institute, Arizona State University; Department of Kinesiology, University of \\ Illinois; Department of Kinesiology, Kansas State University; and College of Health and Human Performance, \\ Oregon State University
}

\begin{abstract}
The influence of maximal exercise testing on state anxiety was examined in three separate studies. Highly trained male distance runners (Study $1, n=12$ ) as well as college students with average (Study $2, n=16$ ) and below average (Study 3, $n=32$ ) physical fitness levels completed graded maximal exercise tests. This last group was also randomly assigned to either a control or an 8 week training programme in order to determine the effect of increased fitness on the psychological responses to maximal exercise testing. Physical fitness was determined by the measurement of maximal oxygen uptake. State anxiety (State-Trait Anxiety Inventory) was assessed before and from 2-15 min following exercise. It was found that the state anxiety responses to maximal exercise testing were not influenced by re-testing or by 8 weeks of endurance training. Across the three study groups, the anxiety response was variable during the first $5 \mathrm{~min}$ following exercise testing; increases, decreases and no changes in anxiety were observed when compared to pre-exercise levels. The anxiety response to maximal exercise appeared to be dependent on the pre-exercise anxiety levels as well as the timing of the post-exercise assessments. It is concluded that maximal exercise testing can be associated with negative mood shifts during the first $\mathbf{5} \mathrm{min}$ after exercise; however, this response is transitory and followed by positive mood shifts 10-15 min following such tests.
\end{abstract}

Keywords: exercise testing, mood, state anxiety

Physiological responses to maximal exercise tests have been extensively described ${ }^{1}$; however, little is known about the psychological aspects of these procedures. Given the widespread use of maximal exercise testing in a variety of medical and health settings there is a need for additional empirical data concerning the psychological aspects of maximal exercise tests. Only a few investigators have examined psychological responses to maximal exercise testing. One report involved 28 adult males who performed both a walking and a running maximal exercise test ${ }^{2}$. It was found that during and immediately following maximal treadmill exercise, anxiety was significantly increased compared to pre-exercise levels. This anxiety increase was followed by a

Address for correspondence: Patrick J. O'Connor, PhD,

Department of Exercise Science, University of Georgia, Athens, Georgia 30602, USA subsequent return to pre-exercise levels within $5 \mathrm{~min}$ post-exercise. In a separate investigation, anxiety, depression and hostility levels were examined in nine adult males immediately before and $5 \mathrm{~min}$ following a maximal bicycle ergometry test ${ }^{3}$. It was found that none of the three psychometric variables assessed was affected by the exercise. The results of these investigations contrast with research findings which have consistently demonstrated that submaximal exercise is associated with mood improvements such as reduced anxiety ${ }^{4}$. Thus, the currently available research suggests that psychological responses to maximal exercise may differ from those which occur with submaximal exercise. If this were true, it would have important implications for causal mechanisms which have been proposed to account for anxiety reductions following acute exercise. Specifically, putative explanations for affective changes with a single bout of exercise have centred on why mood improvements occur. However, if mood is not improved following maximal exercise tests, this finding would have to be incorporated into models which attempt to explain the psychological consequences of exercise behaviour. For this reason, the present report summarizes the results of three separate studies in which the influence of maximal exercise testing on state anxiety has been examined.

\section{Method}

\section{Subjects}

Participants were college student volunteers who responded to solicitations made in classes, on bulletin boards and in a campus newspaper. Every subject read and signed an informed consent document which had been approved by the University's Human Subjects Institutional Review Board.

\section{Procedure}

Maximal exercise tests

Different testing protocols were used in the three studies because each investigation had a separate primary purpose which was unrelated to the maximal exercise testing. Nevertheless, all the maximal exercise tests followed established exercise testing guidelines 5 . Heart rate was monitored continuously during 
the exercise tests using either a 3-lead EKG system or a Quantum XL heart rate monitoring system which uses telemetry to transmit the heart rate from two chest electrodes to a small microcomputer worn at the wrist. Expired gases were monitored using an on-line open spirometry system. Oxygen uptake $\left(\dot{V}_{\mathrm{O}_{2}}\right), \mathrm{CO}_{2}$ production, and ventilatory volume were assessed continuously during exercise.

Every subject performed a maximal exercise test as operationally defined by the achievement of at least two of the following three criteria: (1) a levelling off of $\dot{V}{ }_{2}$ with increasing work (i.e. maximal oxygen uptake $\left.\left[\dot{V}_{\mathrm{O}_{2 \max }}\right]\right) ;(2)$ a heart rate within 10 beats of the subject's age-predicted maximal heart rate (i.e. 220 minus age); or (3) a maximal perceived exertion level. Ratings of perceived exertion (RPE) were made using a 15-point scale in which the ratings can range from $6-20^{6}$. An exercise effort is considered to be maximal when ratings of 18 or higher are obtained ${ }^{5}$.

\section{Psychometric measures}

State anxiety assessments were made using one of three related instruments. In study 1 the 20 -item state portion (Form Y1) of the State-Trait Anxiety Inventory (STAI) was utilized ${ }^{7}$. A different form of the STAI (Form X1; also 20-items) was employed in Study $3^{8}$. In Study 2 a 10-item version of the $\mathrm{STAI}^{9}$ was used in order to facilitate repeated measurements over a short time period (i.e. $2 \mathrm{~min}$ ). Spielberger and colleagues have recommended this 10 -item scale when multiple measurements are to be made over a brief time period, and this shorter form has been shown to be highly correlated $(r=0.95)$ with the full, 20 -item STAI. The 10-item scale consists of the following items from the 20-item (Y-1) scale: $1,3,5,7,9,12,13,15,17$ and 19. Most subjects complete the 10 -item scales in $1 \mathrm{~min}$ and the 20 -item scale in 2-3 min. Each of the state anxiety scales are highly intercorrelated and have well-demonstrated content, convergent, discriminant and factorial validities. Normative data are also available for each instrument. In every study the subjects responded to the standard instructional set: 'how you feel right now, that is, at this moment'.

\section{Statistical analyses}

The primary analyses involved multivariate analysis of variance (MANOVA) with repeated measures. When significant effects were observed the location of mean differences were determined using Tukey's post hoc procedure. Effect sizes $\left[\left(\mathrm{M}_{\text {pre }}-\mathrm{M}_{\text {post }} / \mathrm{SD}_{\text {pre }}\right]\right.$ were calculated to determine the magnitude of the pre- to post-exercise effects. Descriptive statistics in the text have been reported as means(s.d.).

\section{Study 1}

The first study was designed to determine the effects of maximal exercise testing on state anxiety levels in physically fit athletes. Highly trained distance runners were of interest because they would be able to achieve a high absolute power output, and because they were accustomed to performing bouts of high intensity exercise.

\section{Method}

\section{Subjects}

Twelve highly trained male distance runners were recruited to participate. The group was characterized by a mean(s.d.) age, height and weight of $28.0(3.7 \mathrm{yrs}), 179.9(3.6 \mathrm{~cm})$ and $70.7(5.9 \mathrm{~kg})$, respectively.

\section{Procedure}

Each of the subjects completed four maximal treadmill running tests on separate days. Two testing protocols were used. The first protocol involved changes in speed or grade in $3 \mathrm{~min}$ stages while the second protocol used $5 \mathrm{~min}$ stages. The subjects performed both protocols twice, and the testing order was randomized using a Latin squares design. This design allowed for a comparision between the two test protocols as well as a determination of the reliability of the state anxiety responses to both of the maximal exercise testing procedures.

The subjects began each test by running at $6 \mathrm{mph}$ on a $0 \%$ grade. During the initial 5 stages the speed was increased by $1 \mathrm{mph}$ per stage. Thereafter, the grade was increased by $2.5 \%$ per stage until a maximal effort had been achieved. Next, a $10 \mathrm{~min}$ recovery period ensued. Initially, the subjects jogged or walked for 2-3 min until their heart rate slowed to less than $120 \mathrm{~b} \mathrm{~min}^{-1}$. The subjects then sat for the remainder of the recovery period. Following the recovery period the subjects warmed up again by running for $2 \mathrm{~min}$ at a moderate intensity. The subjects then exercised for $1-2 \mathrm{~min}$ at a stage above the highest achieved during the just completed maximal effort (i.e. same speed, but a $2.5 \%$ higher grade). This 'supramaximal' exercise was performed to ensure that a true physiological maximum (i.e. $\left.\dot{V} \mathrm{O}_{2 \max }\right)$ had been obtained. State anxiety was assessed starting $15 \mathrm{~min}$ before and $5 \mathrm{~min}$ after the exercise tests.

\section{Results}

The psychological and physiological responses to the maximal exercise tests are presented in Table 1 . As expected the exercise duration was longer $(M=$ $9.3 \mathrm{~min}$ ) during the tests which used $5 \mathrm{~min}$ stages

Table 1. Psychobiological responses to maximal treadmill running

\begin{tabular}{|c|c|c|c|c|}
\hline & \multicolumn{2}{|c|}{3 min stages } & \multicolumn{2}{|c|}{5 min stages } \\
\hline & \multicolumn{2}{|c|}{ Trial } & \multicolumn{2}{|c|}{ Trial } \\
\hline & 1 & 2 & 1 & 2 \\
\hline $\begin{array}{l}\text { Exercise time } \\
\text { (min) }\end{array}$ & 19.5(3.9) & $19.4(3.3)$ & $28.7(5.2)$ & 28.8(5.9) \\
\hline $\begin{array}{l}\mathrm{VO}_{2} \max \\
(\mathrm{ml} \mathrm{kg} \mathrm{min}\end{array}$ & $66.7(5.8)$ & $66.1(4.1)$ & $66.7(5.4)$ & $64.9(4.4)$ \\
\hline $\begin{array}{l}\text { Heart rate max } \\
\left(b \min ^{-1}\right)\end{array}$ & 186.2(7.0) & 185.7(7.0) & $186.9(5.5)$ & $184.5(5.4)$ \\
\hline State anxiety & & & & \\
\hline $\begin{array}{l}\text { Pre-exercise } \\
\text { Post-exercise }\end{array}$ & $\begin{array}{l}30.6(5.9) \\
28.4(6.8)\end{array}$ & $\begin{array}{l}33.4(10.3) \\
31.9(11.4)\end{array}$ & $\begin{array}{l}31.7(7.3) \\
28.3(5.6)\end{array}$ & $\begin{array}{l}29.8(9.0) \\
27.9(7.5)\end{array}$ \\
\hline $\begin{array}{l}\text { Post-exercise } \\
\text { Effect size }\end{array}$ & $\begin{array}{l}28.4(6.8) \\
0.35\end{array}$ & 0.14 & $\begin{array}{l}28.3(3.6) \\
0.52\end{array}$ & 0.23 \\
\hline
\end{tabular}

All values are mean(s.d.) 
compared to the $3 \mathrm{~min}$ stage tests. Repeated measures MANOVAs revealed that none of the psychological or physiological variables assessed differed across the four maximal exercise trials: pre-test anxiety $F(3,9)=1.3$; post-test anxiety $F(3,9)=3.3$; maximal heart rate $F(3,9)=1.1$; and $V_{\mathrm{O}_{2} \max } F(3,9)=$ 1.3 , all $P_{s}>0.05$. The magnitude of the pre- to post-exercise changes in state anxiety (i.e. the effect sizes) were small, and the change scores did not differ across the four maximal exercise bouts, $F(3,9)$ $=3.4, P>0.05$.

\section{Discussion}

The average pre-exercise anxiety score across the four trials was 31.4 , and this was approximately $0.5 \mathrm{~s}$.d. below the published norms of 36.5 for both male college students and working adults aged 19-397. This observation of low pre-exercise anxiety is in contrast with a previous demonstration of elevated anxiety prior to intense running exercise ${ }^{10}$. One plausible explanation of the low pre-exercise anxiety level in the present study is that maximal treadmill running may be perceived as being less threatening in highly trained distance runners compared to less fit individuals, and therefore provokes less anxiety. Also, low pre-exercise anxiety results are consistent with the much replicated observation that groups of endurance athletes can be discriminated from "normals' based upon the more positive mood states (e.g. lower anxiety) in the athletes ${ }^{11}$. For these reasons, the observation of a below average level of state anxiety $15 \mathrm{~min}$ prior to a maximal exercise test in highly trained distance runners was not surprising.

The state anxiety responses were found to be replicable across the four exercise bouts. Since both the shorter $(19.5 \mathrm{~min})$ and longer $(28.7 \mathrm{~min})$ protocols were associated with similar non-significant reductions in anxiety following exercise, the findings suggest that the anxiety response to maximal exercise is not strongly linked to exercise duration. Furthermore, the observation that anxiety levels did not change following exercise was consistent with the previous reports showing no change in anxiety compared to baseline when anxiety was assessed $5 \mathrm{~min}$ post-exercise ${ }^{2,3}$. Nevertheless, it has previously been shown that the timing of the post-exercise anxiety assessment has a crucial impact on the anxiety response to exercise that is observed ${ }^{12}$.

\section{Study 2}

Study 2 extended Study 1 by: (1) examining the state anxiety response to maximal exercise testing in individuals who exercise regularly but were less physically fit, and (2) describing the time course of anxiety responses to maximal exercise testing in more detail.

\section{Method}

Subjects

The subjects were 16 male volunteers with a mean(s.d.) age, height, weight and $\dot{V}_{\mathrm{O}_{2}} \max$ of $22.9(2.4 \mathrm{yrs}), \quad 181.8(7.3 \mathrm{~cm}), \quad 78.4(10.7 \mathrm{~kg}) \quad$ and $54.7\left(8.2 \mathrm{ml} \mathrm{kg}^{-1} \mathrm{~min}^{-1}\right)$, respectively. The mean $\dot{V}_{\mathrm{O}_{2} \max }$ value indicates that this group possessed fitness levels which were similar to mean values published for males of this age group ${ }^{1}$.

Procedure

Each subject performed a progressive treadmill running test to maximum. Immediately before as well as 2 and $10 \mathrm{~min}$ following exercise, state anxiety measurements were initiated using the 10-item state anxiety scale. The exercise tests began with a $3 \mathrm{~min}$ warm up stage which consisted of running at $6 \mathrm{mph}$ and $0 \%$ grade. Subsequent stages lasted $2 \mathrm{~min}$, and the first stage involved running at $7 \mathrm{mph}$ and $0 \%$ grade. Thereafter, the speed was held constant and the grade was increased by $2.5 \%$ until volitional exhaustion.

\section{Results}

The total exercise time averaged(s.d.) $11.7(2.8) \mathrm{min}$, and the mean(s.d.) maximal heart rate response was $188.8\left(8.8 \mathrm{~b} \mathrm{~min}^{-1}\right)$. The state anxiety results for Study 2 are illustrated graphically in Figure 1. Repeated measures MANOVA revealed a statistically significant effect across the three trials, $F(1,9)=8.6$, $P<0.004$. Post hoc probes indicated that state anxiety was reduced at both 2 and $10 \mathrm{~min}$ post-exercise compared to the pre-exercise value. There was, however, no significant difference between the two post-exercise values. The effect size of the anxiety reduction was 0.68 at $2-\mathrm{min}$ and 0.98 at $10-\mathrm{min}$ post-exercise.

\section{Discussion}

The significant reduction in state anxiety following the maximal exercise tests in Study 2 is in contrast both with prior published research ${ }^{2,3}$ as well as the results obtained in Study 1. A number of factors might account for the inconsistencies across studies. It is possible, for example, that anxiety reduction following maximal exercise is delayed when individuals achieve absolute workloads that are significantly greater than can be attained by a person possessing an average level of physical fitness. A second possible explanation concerns the preexercise anxiety values. In Study 1 the average

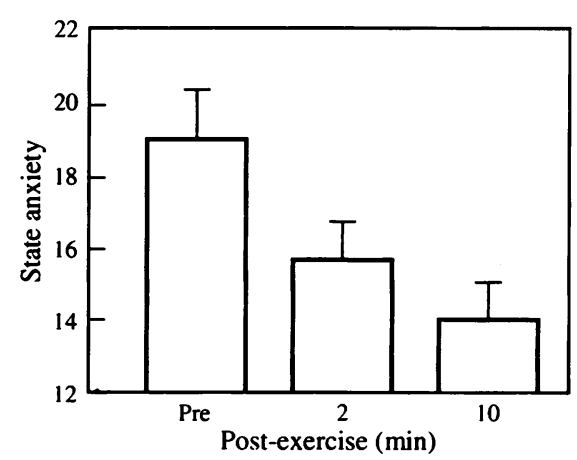

Figure 1. State anxiety before and after maximal treadmill running in 16 male subjects 
pre-exercise anxiety score across the four trials was approximately $0.5 \mathrm{~s}$.d. below the published norms. These relatively low initial values may have limited the possibility for significant post-exercise reductions in state anxiety. In contrast, the mean pre-exercise state anxiety value in Study $2(19.4)$ was 0.25 s.d. above the reported norms (17.9(5.9)) for college aged males ${ }^{9}$. Since the items which constitute the 10-item scale used in Study 2 represent a split-half form of the 20-item STAI used in Study 1, the full scale scores can be approximated by doubling the 10-item scores. When this was done, the mean full scale pre-exercise state anxiety value was estimated at 38.8 . Thus, the participants in Study 2 possessed pre-exercise anxiety estimated to be higher than that for the subjects in Study $1(M=31.4)$.

Since one suggestion from these findings was that pre-exercise anxiety levels may influence whether anxiety reductions occur following exercise, correlations were computed between the pre-exercise anxiety values and the change in anxiety with exercise. It was found that the change in anxiety at $10 \mathrm{~min}$ post-exercise was significantly correlated $(r=$ $0.65)$ with pre-exercise anxiety levels. The correlations at the $2 \mathrm{~min}$ post-exercise period ( $r=0.27$ ) in Study 2 as well as the $5 \mathrm{~min}$ post-exercise period in Study $1(r=0.28)$ did not achieve statistical significance. Thus, the correlational findings are consistent with the notion that those individuals with higher state anxiety prior to maximal exercise testing are more likely to show a decrease in anxiety at the $10 \mathrm{~min}$ post-exercise period only. The results highlight the importance of considering both the preexercise anxiety levels as well as the timing of the post-exercise measurements in understanding anxiety responses to maximal exercise testing.

\section{Study 3}

Study 3 extended the results of the first two studies by: (1) examining state anxiety responses in low-fit individuals, and (2) determining if endurance training would influence the anxiety responses to maximal exercise testing.

\section{Method}

\section{Subjects}

Students were recruited who had been sedentary for a minimum of three months. These subjects were randomly assigned to either a control or an experimental group. Four of the 36 subjects who initially agreed to participate in the study dropped out (one experimental and three control). Thus, complete data were obtained on 17 experimentals (10 females and seven males) and 15 controls (nine females and six males). As expected the randomization process generated groups which did not differ in age, height, weight, $\dot{V}_{2}$ max or state anxiety level. Also as expected, in both the experimental and control groups the males were taller, heavier and had a greater $\dot{V}_{2}$ max than the females.

\section{Procedure}

The control subjects participated in pre- and posttesting sessions only. The experimental subjects completed a total of $40 \mathrm{~min}$ of supervised cycle ergometry (COMBI ergometers), $30 \mathrm{~min}$ of which was at an intensity equal to $60-85 \%$ of their heart rate reserve (5-min warm-up and cool down), 3 days per week for a total of 8 weeks. When a subject missed an exercise session, the individual was contacted and the session was made up. Thus, all subjects completed a total of 24 exercise sessions. Training of this nature in sedentary individuals has previously been shown to result in an increase in fitness of approximately $10 \%{ }^{14}$.

Since the endurance training was performed on bicycle ergometers, the maximal exercise testing involved progressive cycle ergometry. The subjects pedalled at $60 \mathrm{rpm}$ at an initial power output of $100 \mathrm{~W}$ for the males and $50 \mathrm{~W}$ for the females. Every 2 min the resistance was increased by $50 \mathrm{~W}$ for the males and $25 \mathrm{~W}$ for the females until the subjects could no longer continue. State anxiety assessments were made $15 \mathrm{~min}$ before as well as 5 and $15 \mathrm{~min}$ following exercise.

\section{Results}

The $\dot{V}_{2}$ max values confirmed the low-fit nature of the subjects who participated in this study. At the pre-test the females in the experimental group were characterized by a mean(s.d.) $\dot{V}_{2 \max }$ of $27.4(3.6) \mathrm{ml}$ $\mathrm{kg}^{-1} \min ^{-1}$, and the corresponding values for the males were $32.0(6.2)$. These averages did not differ $(P>0.05)$ from corresponding values for the controls, and both females and males were more than 2 s.d. below the estimated population average for college students $^{1}$. Repeated measures MANOVA revealed non-significant main effects (i.e. Group = experimentals and controls; Trials = pre- and post-training) for $\dot{V}_{\mathrm{O}_{2 \text { max }}}$. The Group X Trial interaction for $\dot{V}_{\mathrm{O}_{2} \max }$ revealed an $F$ value $(2,59)$ of 3.6 with an associated $P$ $=0.06$. The mean(s.d.) $V_{\mathrm{O}_{2} \max }$ for the experimental group increased to $33.2(5.1) \mathrm{ml} \mathrm{kg} \mathrm{kmin}^{-1}$ from 29.3(5.2) while the mean(s.d.) $\dot{V} \mathrm{O}_{2 \max }$ of the control group decreased from $30.3(6.9) \mathrm{ml} \mathrm{kg}^{-1} \mathrm{~min}^{-1}$ to a value of $28.6(6.0)$ at the post-testing period.

The descriptive data for state anxiety for the resultant experimental and control groups are presented in Table 2. For state anxiety, significant effects were not observed for the Group, Trial or interaction

Table 2. Anxiety responses to maximal exercise testing before and after training

\begin{tabular}{|c|c|c|c|}
\hline & Pre-exercise & $\begin{array}{l}\text { Post-exercise } \\
\text { (5-min) }\end{array}$ & $\begin{array}{l}\text { Post-exercise } \\
\quad(15-\min )\end{array}$ \\
\hline \multicolumn{4}{|c|}{ Experimentals $(n=17)$} \\
\hline Pre-testing & $30.9(9.7)$ & $36.5(8.3)$ & $30.6(7.7)$ \\
\hline Post-testing & $34.5(12.6)$ & $35.6(12.3)$ & $30.4(10.4)$ \\
\hline \multicolumn{4}{|c|}{ Controls $(n=15)$} \\
\hline Pre-testing & $29.5(6.3)$ & $36.1(9.3)$ & $31.1(8.7)$ \\
\hline Post-testing & $30.2(7.0)$ & $36.9(9.2)$ & $31.5(7.5)$ \\
\hline
\end{tabular}

All values are mean(s.d.) 
effects $(P>0.05)$. For both groups at each trial the maximal exercise testing was associated with significant pre-to-post exercise alterations in state anxiety across the three measurement periods; $F(2,59)=$ 27.0, $P=0.001$. Post hoc tests showed that state anxiety was higher at the $5 \mathrm{~min}$ post-exercise period compared to the other two assessment periods, and that state anxiety assessed $15 \mathrm{~min}$ post-exercise did not differ from the pre-exercise anxiety level.

\section{Discussion}

The $13 \%$ increase in $\dot{V}_{\mathrm{O}_{2} \max }$ associated with training in the experimental group was consistent with the fitness changes expected as a result of an 8 week training programme of the type employed in this study ${ }^{14}$. The primary finding of this investigation was that physical training had no influence on the anxiety responses to maximal exercise testing. It is, of course, possible that more intensive training over a longer period of time might influence acute psychological responses to exercise. However, to our knowledge reports concerning the question of whether chronic physical training influences acute psychological responses to aerobic exercise have not previously been published.

\section{Discussion}

Based on the results of these studies a number of general conclusions can be made regarding the effects of maximal exercise testing on state anxiety. First, maximal exercise can, in some instances, be associated with increased anxiety, but such elevations are transitory in nature. Furthermore, state anxiety was never elevated above pre-exercise levels for more than $5 \mathrm{~min}$ following an exercise test. Secondly, anxiety responses to exercise testing are not influenced by re-testing either over a brief time period (i.e. over 2-10 days in Study 1) or over a longer term (i.e. over 8 weeks with controls in Study 3). Third, a typical physical training programme does not influence anxiety responses to acute maximal exercise testing. This differs from what occurs with many physiological variables. For example, the heart rate response to exercise during maximal exercise will be reduced at any given absolute workload following endurance training ${ }^{1}$. Thus, it appears that the affective responses to maximal exercise testing may not be adaptive to physical training. There is an absence of investigations concerning the effects of chronic exercise on psychological responses to acute exercise and such information would add to our understanding of the relationship between exercise and affect.

A fourth general observation was that state anxiety responses to maximal exercise testing appeared to depend in part on the pre-exercise anxiety level as well as the timing of the post-exercise assessment. When decrements in anxiety are observed 10 to $15 \mathrm{~min}$ following an exercise test, there is some evidence, although admittedly correlational, that the largest decreases occur in subjects who have preexercise anxiety levels above the population norms.
The studies reported here were not aimed at determining the mechanism(s) by which exercise might alter anxiety, nevertheless, the results may have implications for future mechanistic studies. A number of potential explanations have been forwarded to account for the anxiety reductions observed in association with submaximal exercise. These have been previously summarized ${ }^{4,11}$ and they include the following hypotheses: (1) exercise elevates brain levels of endorphins and anxiety reduction may be brought about by the physiological activity of the endorphins within the central nervous system; (2) exercise elevates the temperature of the body and anxiety reduction occurs perhaps because of the effect of the increased temperature on muscle tension, or as a result of the pleasure experienced as the body cools from an adversely high temperature achieved during exercise; and (3) exercise involves taking time out from one's daily schedule and exercise may reduce anxiety by providing individuals with a distraction from the day's cares and worries. While all three of these hypotheses are tenable, they have yet to be integrated with the observation that anxiety can be increased during and within $5 \mathrm{~min}$ following maximal exercise. For example, the distraction hypothesis alone would appear to be unable to account for the increases in anxiety immediately following maximal exercise testing as well as anxiety reduction observed later in the post-exercise period. While it is not new to point out that numerous mechanisms may interact to account for anxiety responses to exercise, it may be of use for researchers to consider how anxiety changes during exercise and the post-exercise period in refining future hypotheses with regards to mechanisms.

These studies were conducted with young, nonanxious adults for whom maximal exercise tests were not contraindicated, and the findings may not generalize to special populations for whom maximal exercise testing is difficult or poses potential physical risks (e.g. children, elderly, cardiac patients). Nevertheless, it is concluded that maximal exercise testing can be associated with negative mood shifts during the first $5 \mathrm{~min}$ after exercise, however, this response is transitory and followed by positive mood shifts 10-20 min following such tests.

\section{Acknowledgements}

Appreciation is expressed to Jon P. Veltri for his expert technical assistance with Study 1 .

\section{References}

1 Astrand P-O, Rodahl K. Textbook of Work Physiology, 2nd Ed. New York, USA: McGraw-Hill, 1977.

2 Morgan WP, Hostman DH, Cymerman A, Stokes J. Exercise as a relaxation technique. Primary Cardiology 1980; 9: 48-57.

3 Goldfarb AH, Hatfield BD, Sforzo GA, Flynn MG. Serum B-endorphin levels during a graded exercise test to exhaustion. Med Sci Sports Exerc 1987; 19: 78-82.

4 Morgan WP. Affective beneficence of vigorous physical activity. Med Sci Sports Exerc 1985; 17: 94-100.

5 American College of Sports Medicine. Guidelines for Exercise Testing and Prescription, 4th ed. Philadelphia, Pennsylvania, USA: Lea \& Febiger, 1991.

6 Borg GAV. Perceived exertion: a note on 'history' and methods. Med Sci Sports Exerc 1973; 5: 104-9. 
7 Spielberger CD, Gorsuch RL, Lushene R, Vagg PR, Jacobs GA. Manual for the State-Trait Anxiety Inventory (Form Y1). Palo Alto, California, USA: Consulting Psychologists Press, 1983.

8 Spielberger CD, Gorsuch RL, Lushene R. Manual for the State-Trait Anxiety Inventory (Form X1). Palo Alto, California, USA: Consulting Psychologists Press, 1970.

9 Spielberger CD, Jacobs G, Crane R, Russell S, Westbery L, Barker L, Johnson E, Knight J, Marks E. Preliminary Manual for the State-Trait Personality Inventory (STPI). Unpublished manual, University of South Florida, 1979.

10 O'Connor PJ, Carda RD, Graf BK. Anxiety and intense running exercise in the presence and absence of interpersonal competition. Intern J Sports Med 1991; 12: 423-6.
11 Morgan WP. Selected psychological factors limiting performance: a mental health model. In: DH Clarke and HM Eckert, eds. Limits of Human Performance. Champaign, Illinois, USA: Human Kinetic, 1985; 70-80.

12 Petruzzello SJ, Landers DM, Hatfield BD, Kubitz KA, Salazar W. A meta-analysis on the anxiety reducing effects of acute and chronic exercise. Sports Med 1991; 11: 143-82.

13 Raglin JS, Morgan WP. Influence of exercise and quiet rest on state anxiety and blood pressure. Med Sci Sports Exerc 1987; 19: 456-63.

14 Pollock ML. The quantification of endurance training programs. In: JH Wilmore, ed. Exercise and Sport Science Reviews. New York, USA: Academic Press, 1973: 155-88. 